\title{
The interference in the suicide ideation of patients with malignant tumors by mental clinical nursing pathway
}

\section{Zhaofen Xu \\ Baoan Chen \\ Guohong Li \\ Wenjun Dai}

Department of Gynaecology, Zhongda Hospital, Southeast University, Nanjing, Jiangsu Province, People's Republic of China
Correspondence: Baoan Chen Department of Hematology, Zhongda Hospital, Medical School, Southeast University, Nanjing 210009, Jiangsu Province, People's Republic of China Tel +86 2583272006

Fax +86258327 20II

Email cba8888@hotmail.com
This article was published in the following Dove Press journal:

Patient Preference and Adherence

4 December 2014

Number of times this article has been viewed

Background: The purpose of this study was to examine the effect of mental clinical nursing pathways on suicidal ideation and life quality of patients with malignant tumors.

Methods: Two hundred patients with malignant tumors were randomly divided into a study group and a control group, with 100 patients in each group. During the treatment, patients in the study group received mental clinical nursing pathway care, while those in the control group were given the usual nursing care, such as timely inspection, nurse's reactions to patient's behavior, and execution of medical orders. Thereafter, the "self-rating idea of suicide scale" and Karnofsky Performance Status (KPS) were used to compare the differences in the suicidal ideation of patients with malignant tumors between the two groups before and after the treatment.

Results: There were no statistical differences in the scores of despair factor, optimistic factor, sleep factor, and cover factor between the two groups before the treatment $(P>0.05)$. After different styles of nursing, the former four factors of patients in the study group were significantly lower than those in the control group $(P<0.01)$, while there were no significant differences in the score of cover factor between the two groups $(P>0.05)$. The KPS of patients receiving mental clinical nursing pathway care was higher than those receiving usual nursing care, and there was a statistical significant difference between the two groups $(P<0.01)$. Interestingly, the patients' suicidal ideation scale was negatively correlated with KPS $(r=-0.29, P<0.05)$.

Conclusion: For individuals diagnosed with a malignant tumor, using a mental health clinical nursing pathway can effectively decrease the degree of suicidal ideation and positively impact the quality of life.

Keywords: mental clinical nursing pathway, suicidal ideation, malignant tumor

\section{Introduction}

In recent years, the incidence of cancer has increased year by year, and the number of new cases will increase from 10 million in 2000 to 15 million in 2020 globally according to World Health Organization statistics. ${ }^{1}$ Along with the gradual deterioration of disease, patients often present with mood disorders, such as depression and anxiety. These negative emotions intertwined with intensive pain, difficulty breathing, loss of appetite, and other physical symptoms, are seriously affecting the quality of life of patients, leading some of them to generate suicidal ideation from time to time. Evidence of other literature has reported that the suicide risk in cancer patients is $1.3-2.8$ times higher than in the normal population, ${ }^{2}$ and advanced cancer patients might have an even higher risk of committing suicide. ${ }^{3}$ In our present study, we implemented a mental clinical nursing pathway for patients with malignant tumors during hospitalization, and used the "self-rating idea of suicide scale" (SIOSS) and Karnofsky Performance 
Status (KPS) for assessing the intervention effects of a mental clinical nursing pathway.

\section{Patients and methods}

\section{Participants}

Two hundred patients with malignant tumors were admitted in the Cancer Center of Zhongda Hospital from February 2011 to December 2013, and all enrolled patients provided written informed consent. Inclusion criteria for patient recruitment were as follows: pathological or cytological diagnosis of patients with malignant tumor (without tumor type restrictions), no previous history of mental illness, voluntary collaborators, and expectation of surviving 3 months or more. The patients were randomly divided into two groups, 100 cases formed the study group and 100 cases formed the control group. During the treatment, patients in the study group were given the mental clinical nursing pathway, while those in the control group were given the usual nursing care, such as timely inspection, nurse's reactions to patient's behavior, and execution of medical orders.

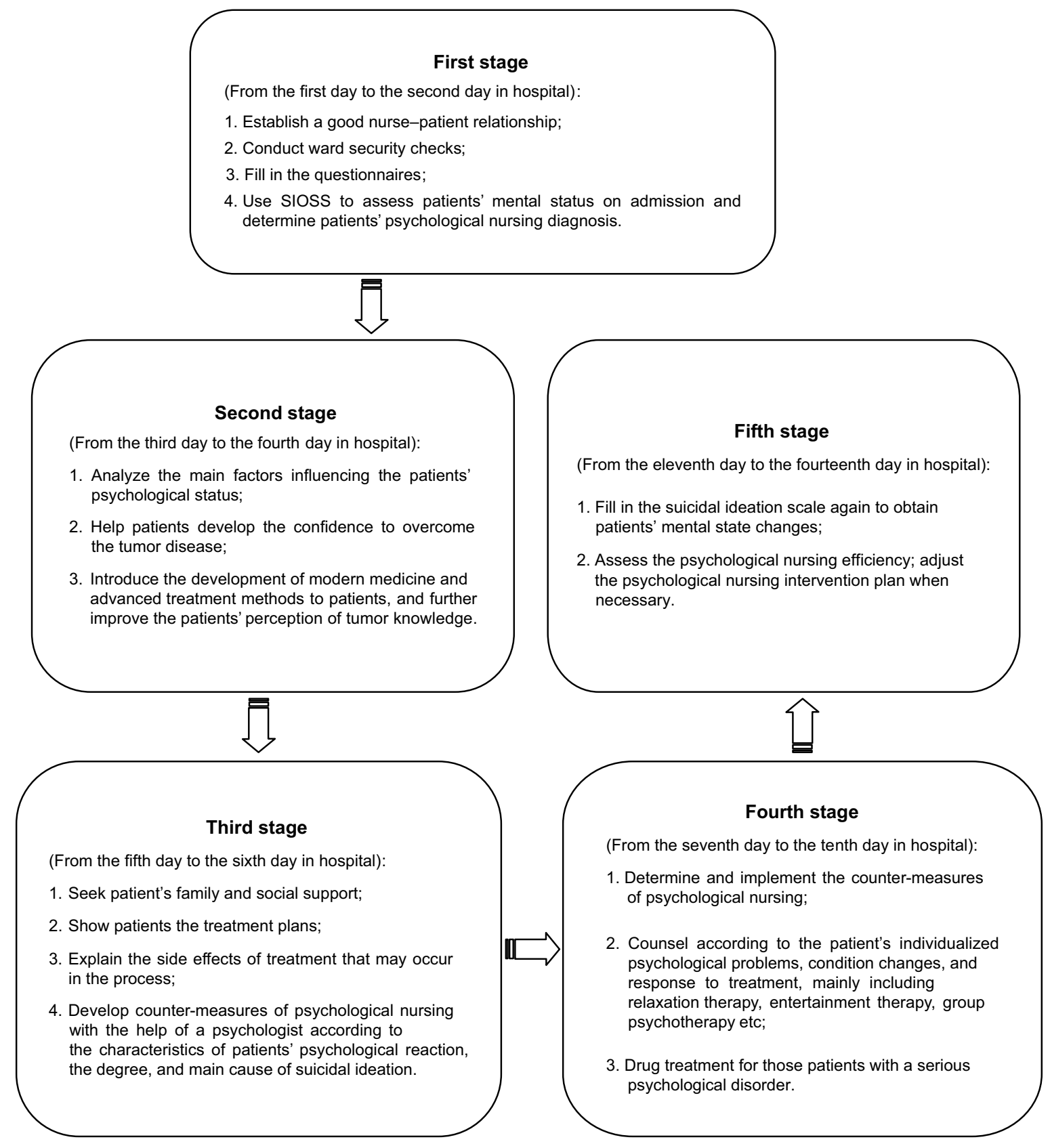

Figure I Stages of mental clinical nursing pathway. Abbreviation: SIOSS, self-rating idea of suicide scale. 


\section{Research tools}

\section{General situation questionnaire}

The questionnaire survey covers general information and tumor specialized subject information. The form includes age, sex, marital status, occupation, level of education, average family income, and source of medical expenses; the latter information includes tumor type, stage, metastasis, duration, informed situation, existence of pain, and previous treatment history.

\section{SIOSS}

Based on the widely used questionnaires of suicidal ideation, such as Beck Depression Inventory, Self-rating Depression Scale, Self-Rating Anxiety Scale, Center for Epidemiologic Studies Depression Scale, Symptom Check List 90, and Minnesota Multiphasic Personality Inventory used in the Editorial Board of Chinese Behavioral Medicine's work, the SIOSS was established by our clinical practice experience. ${ }^{4}$ The scale includes despair factor, optimistic factor, sleep factor, and cover factor, and the total score of suicidal ideation factor was the sum of the four factors. The higher the score, the stronger the suicidal ideation. If the cover factor score was more than four points, it was not reliable to measure. According to the results of statistical tests and our observations, the scale, which had a good validity and reliability, was simple, rapid, effective, and suitable for Chinese self-rating scale screening for suicidal ideation. ${ }^{4}$

\section{KPS}

The scale was proposed firstly in 1948 by Mor et al and mainly used to measure the physical performance status of cancer patients. ${ }^{5}$ KPS is divided into ten levels and has a higher rate of reliability and structural validity. In our present study, it was mainly used to assess cancer patients' quality of life by the intervention of mental clinical nursing pathway.

\section{Methods}

\section{Questionnaire survey procedure and method}

First, professional nurses were trained by the oncology psychiatrist on how to conduct the questionnaire survey, such as the interpretation of each item, language communication skills, and avoidance skills of sensitive issues. Once patients' written informed consent was obtained, questionnaires were given to them and answered independently. General information of the questionnaire, SIOSS, and KPS were recorded on the first day of admission, and then the SIOSS and KPS were recorded again 14 days after admission.

\section{Mental nursing clinical pathway}

The clinical pathway chart was worked out by doctors and nurses, and the psychological care was performed from admission to discharge by the responsible nurse according to the planned route of work program. Implementation of clinical psychological nursing path was mainly done by conversational communication and language communication, and symptomatic medications were given to the patients when necessary under the advice of physicians, such as sedative hypnotic drugs, antidepressants, analgesics, antiemetic drugs, and nutritional support etc. Psychological nursing time required was more than 20 minutes per day during the first week and then more than 20 minutes every 2 days during the second week in hospital. The stages of the mental nursing clinical pathway are shown in Figure 1. In addition, we could identify the patients who urgently needed suicide crisis intervention according to the suicidal ideation score. For these patients, except for the clinical pathway of psychological nursing, we should keep timely contact with patients' families who will care for them every day. Notably, we should also strengthen ward patrols and safety inspections, and keep the patients away from dangerous objects to protect patients' safety and reduce the suicide rate. ${ }^{6}$

\section{Statistical analysis}

Data analysis was performed using SPSS software (version 15.0; SPSS Inc., Chicago, IL, USA). A Student's $t$-test and chi-square test was used to compare SIOSS and KPS between the two groups before and after treatment and Spearman's correlation analysis was applied to analyze the correlation of changes in the SIOSS and KPS score of the study group.

\section{Results Comparison of the suicide ideation scale scores}

There was no statistical difference in the scores of despair factor, optimistic factor, sleep factor, and cover factor between the two groups before treatment $(P>0.05)$. After different styles of nursing, the score of the former four factors in the study group was significantly lower than those in the control group $(P<0.01)$, while there was no significant difference in the scores of cover factor between the two groups $(P>0.05)$ (Table 1).

\section{Comparison of the KPS scores}

The KPS of patients who received mental clinical nursing pathway care was $68 \%$ for 100 score, $14 \%$ for 80 score, 
Table I Comparing the suicide ideation scale before and after the treatment

\begin{tabular}{llllll}
\hline & \multicolumn{2}{l}{ Control group $(\mathbf{n}=\mathbf{1 0 0})$} & & \multicolumn{2}{c}{ Study group $(\mathbf{n}=\mathbf{I 0 0})$} \\
\cline { 2 - 3 } & Before treatment & After treatment & & Before treatment & After treatment \\
\hline Despair factor & $5.04 \pm 3.05$ & $4.13 \pm 2.95$ & $5.10 \pm 3.12$ & $3.5 I \pm 2.86$ \\
Optimistic factor & $0.86 \pm 1.02$ & $0.82 \pm 0.82$ & $0.84 \pm 1.06$ & $0.61 \pm 0.73$ \\
Sleep factor & $2.15 \pm 1.28$ & $2.01 \pm 1.29$ & $2.20 \pm 1.20$ & $1.65 \pm 1.32$ \\
Cover factor & $1.53 \pm 1.31$ & $1.49 \pm 1.29$ & $1.54 \pm 1.30$ & $1.45 \pm 1.28$ \\
Total score & $9.58 \pm 3.93$ & $8.45 \pm 3.65$ & $9.68 \pm 3.87$ & $7.22 \pm 3.46$ \\
\hline
\end{tabular}

Note: Data are presented as mean \pm standard deviation.

$6 \%$ for 70 score, $6 \%$ for 40 score, $2 \%$ for 20 score, and $4 \%$ for 0 score, while those in the control group was $34 \%$ for 100 score, $21 \%$ for 80 score, $16 \%$ for 70 score, $8 \%$ for 40 score, $13 \%$ for 20 score, and $8 \%$ for 0 score. The KPS score of the study group was higher compared to the control group, and there was a statistical significance between the two groups $(P<0.01)$ (Table 2$)$.

\section{Correlation of patients' suicidal ideation with KPS}

The result of Spearman's correlation analysis suggests that the patients' suicidal ideation was negatively correlated with KPS $(r=-0.29, P<0.05)$.

\section{Discussion}

According to World Health Organization data, the loss of the disability-adjusted life year caused by suicide and self-injury in the People's Republic of China in 1998 was 8.837 million, which accounts for $4.2 \%$ of the total disease burden. As we know, the indicator only assesses the direct health burden caused by individual death or self-injury, and does not include the serious and continuous negative psychological impact caused by suicide on victims' family members and friends. Because of the stimulation of negative life events and physical discomfort including long-term pain, difficulty in breathing, and loss of appetite, suicidal ideation is particularly prominent in cancer patients. Therefore, preventing suicidal behavior in patients with malignant

Table 2 The Karnofsky Performance Status score comparison before and after the treatment

\begin{tabular}{lll}
\hline Score & Control group (\%) & Study group (\%) \\
\hline 100 & 34 & 68 \\
80 & 21 & 14 \\
70 & 16 & 6 \\
40 & 8 & 6 \\
20 & 13 & 2 \\
0 & 8 & 4 \\
\hline
\end{tabular}

tumors is one of the most important tasks for oncology clinical staff.

Suicide is not only the result of interaction of many factors such as psychological, social, and biological, but also a product of interaction between personal qualities and stress under the control of mutual growth along with the decline of risk factors and protective factors. Several studies have identified increased suicide rates among patients with cancer, ${ }^{6-10}$ who are at greater risk of suicidal ideation than the general population. ${ }^{11}$ Thus, a targeted solution to psychological crisis factors such as the increase in the number of social support systems, the improvement of the patient's ability to solve problems, and the development of their courage and selfconfidence, are effective measures to reduce suicide risk in patients with malignant tumors. ${ }^{6,12,13}$ Due to the special nature of nursing work, the nurses in the hospital usually spend more time with patients than other workers, so they have obvious advantages in psychological treatment of patients in daily work. But there are still a number of problems concerning psychological care, such as the lack of objective and effective psychological assessment, the lack of specific and common standardized care plans and procedures, unclear psychological nursing diagnosis, and lower feasibility of implementation of psychological nursing. ${ }^{14}$ Early detection and treatment are the best ways to prevent suicidal ideation and suicide attempts. In the present study, SIOSS was used to assess the psychological state of cancer patients and a clinical application pathway theory was used to develop a detailed and standardized psychological care pattern to improve the initiative of nursing work, enhance the comparability and operability of the job, and reduce the randomness, aimlessness, and unnecessary duplication of nursing processes. Notably, the application of a mental clinical nursing pathway in our present study could regulate malignant tumor patients' nursing care, identify suicide risks as soon as possible, implement timely intervention measures, improve the degree of suicidal ideation in patients, and reduce the suicide rate in hospitalized patients with malignant tumors. Importantly, the result of the 
Spearman's correlation analysis suggests that the patients' suicidal ideation was negatively correlated with KPS. The results of KPS showed that the application of a mental clinical nursing pathway could reduce patients' suicidal ideation and significantly improve the patients' quality of life as well as the medical treatment.

As we know, the idea of a mental clinical nursing pathway in clinical work has not yet been universal, and psychological changes during different periods in patients with tumors are larger. Therefore, a mental clinical nursing pathway still needs to be constantly adjusted and revised to improve the practicality and normativity of psychological intervention. In addition, suicide is the result of multiple factors' interaction. Evidence in other literature suggests that depression and anxiety, and advanced tumor pain and dysfunction, are the two main causes of cancer patients' suicide or suicidal tendencies. ${ }^{15}$ Additionally, if a patient has a comorbid psychiatric disorder, it may be difficult to find a medication that addresses both the psychiatric disorder and suicidal ideation. Thus, it is very important for medical workers to treat the primary disease, especially pain control and recovery of function in patients with malignant tumors. In particular, additional drug therapy is necessary for those patients with severe mental disorders, and suicide crisis intervention should also be adopted to reduce the incidence of suicide.

\section{Acknowledgments}

This work was financially supported by Key Department of Jiangsu Medicine (2012-12).

\section{Disclosure}

The authors report no conflicts of interest in this work.

\section{References}

1. World Health Organization, Department of Chronic Diseases and Health Promotion. National cancer control programs: policies and managerial guidelines (2nd edition). Health and Development Networks. 2002.

2. Tanaka H, Tsukuma H, Masaoka T, et al. Suicide risk among cancer patients: experience at one medical center in Japan, 1978-1994. Jpn J Cancer Res. 1999;90(8):812-817.

3. Akechi T, Okuyama T, Sugawara Y, Nakano T, Shima Y, Uchitomi Y Suicidality in terminally ill Japanese patients with cancer. Cancer. 2004;100(1):183-191.

4. Editorial Board of Chinese Behavioral Medicine. Behavioral medicine inventory manual. Beijing: The Chinese Medicine Electronic Audio and Video Publishing House; 2005.

5. Mor V, Laliberte L, Morris JN, Wiemann M. The Karnofsky Performance Status Scale. An examination of its reliability and validity in a research setting. Cancer. 1984;53(9):2002-2007.

6. Innos K, Rahu K, Rahu M, Baburin A. Suicides among cancer patients in Estonia: A population based study. Eur J Cancer. 2003;39(15): 2223-2228.

7. Yousaf U, Christensen ML, Engholm G, Storm HH. Suicides among Danish cancer patients, 1971-1999. Br J Cancer. 2005;92(6): 995-1000

8. Misono S, Weiss NS, Fann JR, Redman M, Yueh B. Incidence of Suicide in Persons With Cancer. J Clin Oncol. 2008;26(29):4731-4738.

9. Robinson D, Renshaw C, Okello C, Moller H, Davies EA. Suicide in Cancer Patients in South East England from 1996 to 2005: a Populationbased Study. Br J Cancer. 2009;101(1):198-201.

10. Walker J, Waters RA, Murray G, et al. Better off dead: suicidal thoughts in cancer patients. $J$ Clin Oncol. 2008;26(29):4725-4730.

11. Moller HJ. Suicide, suicidality and suicide prevention in affective disorders. Acta Psychiatr Scand Suppl. 2003;(418):73-80.

12. Oquendo MA, Galfalvy H, Russo S , et al. Prospective study of clinical predictors of suicidal acts after a major depressive episode in patients with major depressive disorder or bipolar disorder. Am J Psychiatry. 2004;161(8):1433-1441.

13. Brent DA, Oquendo M, Birmaher B, et al. Peripubertal suicide attempts in offspring of suicide attempters with siblings concordant for suicidal behavior. Am J Psychiatry. 2003;160(8):1486-1493.

14. Liu XH, editor. Nursing Psychology. 2nd ed. Shanghai: Second Military Medical University Press. 1998;282.

15. Fishbain DA. Predictive factors for suicidal ideation in patients with unrespectable lung carcinoma. A 6-month follow-up study. Cancer. 2003;97(12):3127-3129.
Patient Preference and Adherence

\section{Publish your work in this journal}

Patient Preference and Adherence is an international, peer-reviewed, open access journal that focuses on the growing importance of patient preference and adherence throughout the therapeutic continuum. Patient satisfaction, acceptability, quality of life, compliance, persistence and their role in developing new therapeutic modalities and compounds to optimize

\section{Dovepress}

clinical outcomes for existing disease states are major areas of interest for the journal. This journal has been accepted for indexing on PubMed Central. The manuscript management system is completely online and includes a very quick and fair peer-review system, which is all easy to use. Visit http://www. dovepress.com/testimonials.php to read real quotes from published authors. 